\title{
Screening migrants for tuberculosis and latent TB infection: the reward will come later
}

\author{
Jean-Pierre Zellweger \\ Affiliation: Swiss Lung Association, Bern, Switzerland. \\ Correspondence: Jean-Pierre Zellweger, Swiss Lung Association, Chutzenstrasse 10, 3007 Bern, Switzerland. \\ E-mail: zellwegerjp@swissonline.ch
}

@ERSpublications

Screening migrants for TB may contribute to the global decrease expected by the End TB Strategy http://bit.ly/2kdVAtE

Cite this article as: Zellweger J-P. Screening migrants for tuberculosis and latent TB infection: the reward will come later. Eur Respir J 2019; 54: 1901719 [https://doi.org/10.1183/13993003.01719-2019].

In the majority of industrialised countries, migrants represent a large proportion of all cases of tuberculosis (TB) notified and the prospect of TB elimination, even in low-incidence countries, is largely dependent of the possibility of controlling TB in migrants. Most countries have therefore implemented procedures for screening migrants who may be carriers of active TB or, more rarely, for the detection and preventive treatment of latent tuberculosis infection (LTBI). The ideas behind these procedures are to protect the local population from the import of a potential transmissible disease and to prevent the emergence of TB months or years after entry into the country of settlement. The screening procedures are very variable, from the "staircase test", that aimed to detect TB by listening to coughing migrants in Ellis Island, to the systematic chest radiography, questionnaires or interferon- $\gamma$ release assay (IGRA) blood tests used in many countries [1]. Screening for TB may be performed before entry into the country, at the border or after settlement. There is still a debate on the cost-effectiveness of these different procedures [2].

In the current issue of the European Respiratory Journal, VILLA et al. [3] report the experience gathered in Milan, Italy, by the Health Protection Agency and the Regional TB Reference Centre. Between 2016 and 2017, 360000 migrants entered in Italy, most of them from countries with a high TB incidence. Migrants hosted in reception centres in the Milan area were screened for active TB using a questionnaire (integrating risk factors from the country of origin, prior $\mathrm{TB}$ exposure or treatment and current symptoms) and for LTBI using a tuberculin skin test (TST). Migrants with a positive response to the questionnaire or to the TST had a clinical examination including a chest radiograph. Migrants with a positive TST and a normal chest radiograph and aged $<35$ years had a blood test (IGRA) for confirmation of the infection and were offered preventive treatment. Children below the age of 5 years with a positive TST were offered preventive treatment without further screening tests.

Within the 2-year period of observation, 5324 asylum seekers were screened, 270 of them (5.1\%) were positive based on the questionnaire and 2298 (43.2\%) had a positive TST. After clinical and radiological examination, 69 cases of active TB were diagnosed and treated, representing $1.3 \%$ of the total number of asylum seekers screened (1236 cases per 100000) and 875 were eligible for LTBI treatment. The outcome of treatment of active TB was successful in $83.8 \%$ and the completion rate of preventive treatment was $94.3 \%$. 
In themselves, these data are very satisfactory and demonstrates that the screening system was able to detect active TB in a large group of migrants and treat them successfully. Interestingly, the authors did not perform a systematic chest radiograph in all migrants (as implemented in many countries), but selectively in those with symptoms suggestive of $\mathrm{TB}$, a positive questionnaire on risk factors (including origin from a high-burden country) or signs of LTBI. Furthermore, the authors claim that the treatment of LTBI prevented the emergence of a similar number of TB cases, which would develop without intervention within the next months or years in this mobile and hard-to-reach population, in the city of Milan or in another location.

In spite of these satisfactory results, the study also demonstrates some of the difficulties of all screening procedures implemented in Western countries and which explain why the procedures are so diverse between countries, without any consensus on the optimal practice $[4,5]$.

First, the screening was performed on average 6 months after entry into Italy, leaving open the possibility of transmission of active $\mathrm{TB}$ in the meantime to other migrants, caregivers or the local population (although this seems to occur very rarely). Interestingly, 16 out of 69 cases diagnosed with active TB had no symptoms (and a negative score on the questionnaire screening), but only a positive TST. These cases were apparently discovered by the clinical examination and chest radiography, demonstrating that a clinical questionnaire may not be sensitive enough to detect active TB or that the communication between the examiners and the asylum seekers was not optimal. As the migrants with clinical problems had free access to healthcare any time, one can wonder how much the systematic questionnaire contributed in the detection of the other cases that had clinical symptoms and who were assumed to seek help at some time.

Second, the procedure was implemented in a population originating mainly from Sub-Saharan Africa, with a very high incidence rate of TB. In countries experiencing immigration flows from regions of the world with a lower incidence rate of TB (for instance the Middle East), the yield of screening procedures is much lower and the cost-effectiveness of any systematic screening procedure is doubtful $[6,7]$, unless differential screening by country of origin is performed, which may be regarded as discriminatory.

Screening for LTBI and prescription of a preventive treatment with the aim of avoiding future cases of TB is much debated. As the proportion of asylum seekers with a positive test result for LTBI (TST or IGRA) is high (between one quarter and one half depending the origin of the migrants), and the infection may have been acquired long before the immigration, implying that the risk of TB reactivation is low, the number needed to treat to prevent one single case of TB in the future may be regarded as prohibitive. However, restricting screening and preventive treatment to the population groups with the highest risk (children and young adults from high-burden countries) is considered cost-effective [8,9], and studies have confirmed the impact of such a policy on the number of future cases of TB [10,11]. As the majority of migrants screened in Milan were young adults, and originated from high-burden regions, the policy can be considered as cost-effective. Confirmation of the positive TST test results by an IGRA decreased by half the number of asylum seekers eligible for preventive treatment.

Finally, a large proportion of migrants escaped the screening system, which is not surprising, given the fact that the migrants had to visit two different places for initial screening and for further examination if found positive. As the authors mention, concentrating all activities in one single place and avoiding transfers and delays in the further examination could decrease the number of cases lost to follow-up.

The favourable outcome of treatment for active TB and the high proportion of completion of preventive treatment is to be acknowledged and represents a small but significant contribution to the decrease in the number of expected cases of TB. The reward will be for the future.

Conflict of interest: J-P. Zellweger reports personal fees for lectures from Qiagen, during the conduct of the study.

\section{References}

1 Dara M, Gushulak BD, Posey DL, et al. The history and evolution of immigration medical screening for tuberculosis. Expert Rev Anti Infect Ther 2013; 11: 137-146.

2 van der Werf MJ, Lonnroth K. Pre-entry, post-entry, or no tuberculosis screening? Lancet Infect Dis 2014; 14: $1171-1172$.

3 Villa S, Codecasa LR, Faccini M, et al. Tuberculosis among asylum seekers in Milan, Italy: epidemiological analysis and evaluation of interventions. Eur Respir J 2019; 54: 1900896.

4 Coker R. Tuberculosis screening in migrants in selected European countries shows wide disparities. Eur Respir J 2006; 27: 801-807.

5 Kunst H, Burman M, Arnesen TM, et al. Tuberculosis and latent tuberculous infection screening of migrants in Europe: comparative analysis of policies, surveillance systems and results. Int J Tuberc Lung Dis 2017; 21: 840-851.

6 Bozorgmehr K, Joggerst B, Wagner $\mathrm{U}$, et al. Yield of tuberculosis screening in asylum-seekers by country of origin: analysis of screening data in a German federal state (2002-2015). Eur Respir J 2017; 49: 1602327. 
7 de Vries G, van Rest J, Meijer W, et al. Low yield of screening asylum seekers from countries with a tuberculosis incidence of $<50$ per 100000 population. Eur Respir J 2016; 47: 1870-1872.

8 Zammarchi L, Casadei G, Strohmeyer M, et al. A scoping review of cost-effectiveness of screening and treatment for latent tubercolosis infection in migrants from high-incidence countries. BMC Health Serv Res 2015; 15: 412.

9 Zenner D, Hafezi $\mathrm{H}$, Potter J, et al. Effectiveness and cost-effectiveness of screening migrants for active tuberculosis and latent tuberculous infection. Int J Tuberc Lung Dis 2017; 21: 965-976.

10 Zenner D, Loutet MG, Harris R, et al. Evaluating 17 years of latent tuberculosis infection screening in north-west England: a retrospective cohort study of reactivation. Eur Respir J 2017; 50: 1602505.

11 Berrocal-Almanza L-C, Harris R, Lalor MK, et al. Effectiveness of pre-entry active tuberculosis and post-entry latent tuberculosis screening in new entrants to the UK: a retrospective, population-based cohort study. Lancet Inf Dis 2019; in press [https://doi.org/10.1016/S1473-3099(19)30260-9]. 